\title{
Tape Market
}

Many lab directors and their schools are faced with the problem of what to do with the master tapes for texts which are no longer used by their school. They can either erase and rebulk the tapes or store them to gather dust. We offer another solution. We will list in the NALLD Journal tapes for sale by schools. Only those sold by the publisher to the school and in their original package will be listed. The following should be observed:

1. Use this form: language, title, author, editor, year, number of reels, number of tracks, cost, selling price (we suggest $\mathbf{5 0 \%}$ discount), person to contact.

2. NALLD and the NALLD Journal accept no responsibility for the transporation between buyer and seller. The quality of the tapes and the method of payment for them should be a matter established between buyer and seller before the transaction is made.

3. The listing in the Journal will be determined by the space available. The NALLD Journal reserves the right to select listings.

\section{FRENCH}

"PAROLE ET PENSÉE"-Lenard, Harper and Row, 1965; 39 basic tapes, 4 testing tapes, 4 supplementary tapes, $33 / 4$ ips, full track. Cost, $\$ 280$, selling price, $\$ 140$.

CONTACT: Dr. Robert H. Price, Director, Department of Foreign Languages, Sam Houston State University, Huntsville, Texas 77340.

"MODERN FRENCH"-Desberg and Kenan, 1964; twenty 7-inch reels; Cost, $\$ 150$; selling price, $\$ 75$.

CONTACT: Professor Keith R. Bell, Modern Languages, MidAmerica Nazarene College, Olathe, Kansas 66061.

\section{GERMAN}

"GERMAN IN REVIEW"-Hubbs, Valentine and Keyes, Robert Macmillan Co., 1966; 3 two-track, 3\$/4 ips, 7-inch reels, $\$ 15$.

CONTACT: Professor Glenn J. Van Treese, P. 0. Box 18, Sweet Briar College, Sweet Briar, Virginia 24595.

"GERMAN THROUGH CONVERSATIONAL PATTERNS"-Rogers and Watkins, Dodd, Mead and Co., 1965; 12 full-track tapes, 3 3/4 ips. Cost, \$175.; selling price, $\$ 88$.

CONTACT: Dr. Robert H. Price, Director, Department of Foreign Languages, Sam Houston State University, Huntsville, Texas 77340. 
"HÖR GUT ZU"-Schmidt, McMillan, 1964; 5 double-track tapes, 3 3\%/ ips. Cost, $\$ 65$; selling price $\$ 32$.

CONTACT: Same as above.

"GERMAN IN REVIEW"-Sparks and Vail, Harcourt, Brace and World, 1967; 5 full-track tapes, $33 / 4$ ips. Cost, \$40; selling price, $\$ 20$.

CONTACT: Same as above.

"PRACTICE AND PROGRESS: A GERMAN GRAMMAR FOR REVIEW AND REFERENCE"-Weimar and Hoffmeister, Ginn and Co., 14 full-track tapes, $33 / 4$ ips. Cost, $\$ 65$; selling price, $\$ 32$.

CONTACT: Same as above.

\section{SPANISH}

"ESPANOL A LO VIVO, LEVEL II"-Hansen and Wilkins, Blaisdell/ Ginn and Co., 1966; 39 single track tapes, $33 / 4$ ips, $\$ 90$.

CONTACT: Cliff Mountain, Director of Foreign Language, Mesa College, Grand Junction, Colorado 81501.

"ON WITH SPANISH! A CONCEPT APPROACH TO SPANISH"-Da Silva and Lovett, 2nd Edition, Harper and Row, 1970; 20 single track tapes, $33 \frac{3}{4}$ ips, $\$ 75$.

CONTACT: Same as above.

"VOCES Y VISTAS: ACTIVE SPANISH FOR BEGINNERS"-Osborne, Tilles, Perez, Harper and Row, 1970, 33 reels, $33 / 4$ ips, full track. Cost, $\$ 250$, selling price, $\$ 125$.

CONTACT: Dr. Robert H. Price, Director, Department of Foreign Languages, Sam Houston State University, Huntsville, Texas, 77340 .

"BEGINNING SPANISH: A CONCEPT APPROACH"-Da Silva, Harper and Row, 1963 and 1968; 32 basic tapes, 5 review and testing tapes, $33 / 4$ ips, full track. Cost, $\$ 400$; selling price, $\$ 200$.

CONTACT: Same as above.

"A REVIEW OF SPANISH"-Eoff, Sherman, Macmillan Co., 1963; four 7-inch, two-track reels, $33 / 4$ ips, $\$ 25$.

CONTACT: Professor Glenn J. Van Treese, P. O. Box 18, Sweet Briar College, Sweet Briar, Virginia 24595.

"BEGINNING SPANISH: A CONCEPT APPROACH"-2nd Edition, Da Silva, Zenia S., Harper and Row, 1968; thirty-two 5-inch reels, $33 / 4$ ips, $\$ 75$.

CONTACT: Same as above. 